\title{
Consensus aware manufacturing service collaboration optimization under blockchain based Industrial Internet platform
}

Yongping Zhang, Pengyuan Zhang, Fei Tao, Yang Liu and Ying Zuo

The self-archived postprint version of this journal article is available at Linköping University Institutional Repository (DiVA):

http://urn.kb.se/resolve?urn=urn:nbn:se:liu:diva-16015.5

N.B.: When citing this work, cite the original publication.

Zhang, Y., Zhang, P., Tao, F., Liu, Y., Zuo, Y., (2019), Consensus aware manufacturing service collaboration optimization under blockchain based Industrial Internet platform, Computers \& industrial engineering, 135, 1025-1035. https://doi.org/10.1016/j.cie.2019.05.039

Original publication available at:

https://doi.org/10.1016/j.cie.2019.05.039

Copyright: Elsevier

http://www.elsevier.com/

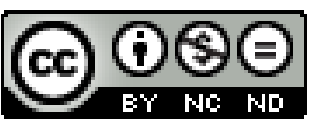




\section{Accepted Manuscript}

Consensus aware manufacturing service collaboration optimization under blockchain based Industrial Internet platform

Yongping Zhang, Pengyuan Zhang, Fei Tao, Yang Liu, Ying Zuo

PII:

$$
\text { S0360-8352(19)30312-2 }
$$

DOI: https://doi.org/10.1016/j.cie.2019.05.039

Reference:

$$
\text { CAIE } 5882
$$

To appear in:

$$
\text { Computers \& Industrial Engineering }
$$

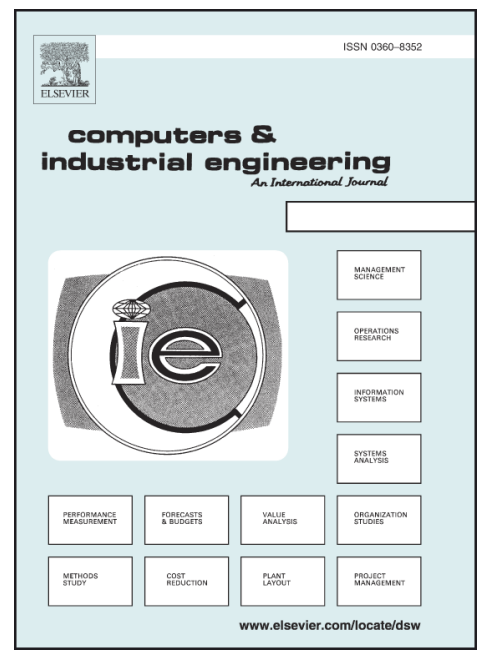

Received Date: 3 January 2019

Revised Date: $\quad 29$ April 2019

Accepted Date: $\quad 30$ May 2019

Please cite this article as: Zhang, Y., Zhang, P., Tao, F., Liu, Y., Zuo, Y., Consensus aware manufacturing service collaboration optimization under blockchain based Industrial Internet platform, Computers \& Industrial Engineering (2019), doi: https://doi.org/10.1016/j.cie.2019.05.039

This is a PDF file of an unedited manuscript that has been accepted for publication. As a service to our customers we are providing this early version of the manuscript. The manuscript will undergo copyediting, typesetting, and review of the resulting proof before it is published in its final form. Please note that during the production process errors may be discovered which could affect the content, and all legal disclaimers that apply to the journal pertain. 


\title{
Consensus aware manufacturing service collaboration optimization under
}

\section{blockchain based Industrial Internet platform}

\author{
Yongping Zhanga ${ }^{\mathrm{a}}$ Pengyuan Zhang ${ }^{\mathrm{a}}$, Fei Tao ${ }^{\mathrm{a}, *}$, Yang Liu ${ }^{\mathrm{b}, \mathrm{c}}$ and Ying Zuo \\ ${ }^{a}$ School of Automation Science and Electrical Engineering, Beihang University, Beijing, 100191, China \\ ${ }^{\mathrm{b}}$ Department of Management and Engineering, Linköping University, SE-581 83 Linköping, Sweden \\ ' Department of Production, University of Vaasa, 65200 Vaasa, Finland
}

Address for Corresponding author: Prof. Fei Tao, School of Automation Science and Electrical Engineering, Beihang University, Beijing 100191, P. R. China.

E-mail: ftao@buaa.edu.cn

Acknowledgments: This work is financially supported in part by National Natural Science Foundation of China (NSFC) under Grant 51875030, National Key Research and Development Program of China (No.2016YFB1101700), and Beijing Science and Technology Project (No. Z181100003118001). Sincere appreciation is extended to the editors and reviewers of this paper for their helpful comments. 


\title{
Consensus aware manufacturing service collaboration optimization under blockchain based Industrial Internet platform
}

\begin{abstract}
To realize collaboration among distributed enterprises, manufacturing service collaboration on Industrial Internet platform is an efficient method. However, the low degree of participation resulted by the sense of distrust, dissatisfaction, and insecurity hinders the widely application of Industrial Internet platform. Therefore, a secure, trustworthy, and multi-user satisfied manufacturing service collaboration method is in urgent need. A blockchain based platform could be utilized to support collaboration among distributed participants to complete trustworthy transactions. In addition, in order to satisfy multiple users, there should be a suitable collaboration mechanism that allows interest-independent participants to fulfil their short/long-term expectations and guide them to a consensus. Based on the establishment of the underlying data and network layer of the blockchain, the collaboration optimization of manufacturing services based on consensus is proposed. By utilizing Memetic algorithm, both the long-term utility of providers and the short-term utility of consumers are combined to choose the optimal providers for the tasks. The providers are selected with higher satisfaction degree of consumers.
\end{abstract}

KEYWORDS Long/short-term utility; Manufacturing service collaboration; Blockchain; Industrial Internet platform; Memetic algorithm

\section{Introduction}

With the advent of sharing economy, the environment that manufacturing companies must face has undergone tremendous changes. They will be encountered with the uncertainty of consumers, the shortening of product life-span and the more complexity of the product structure. Hence, it is difficult for a manufacturing enterprise to meet the diversified needs or accomplish the large-scale tasks. As the widely use of new generation information technologies in manufacturing, the collaboration of smart manufacturing services which are encapsulated by the physical resources and capabilities is an efficient and convenient way to fulfil manufacturing tasks (Tao, Cheng, Xu, Zhang, \& Li, 2014; Tao, \& Qi, 2017).

The Industrial Internet, which refers to the integration of complex physical machinery with networked sensors and software, creates a new way for realizing smart manufacturing. Industrial Internet platform based manufacturing service collaboration promotes the distributed manufacturing resources and capabilities sharing and achieving task-performing. Various Industrial Internet platforms, such as GE Predix, ABB Ability, Siemens MindSphere and PTC ThingWorx emerge, through which manufacturing service transactions can be completed by providers and consumers. However, the low activity of the users hinders the widely application among enterprises. Specifically, before joining the platform, users lack trust in the platform or are unwilling to disclose enterprise data. The trustworthiness of the information storing on the platform is essential for consumers, providers and the platform operator for manufacturing service collaboration (Pouryazdan, Fiandrino, Kantarci, Kliazovich, Soyata \& Bouvry. 2017). After joining the platform, the main reason that hinders the collaboration is e.g. the expected profit cannot be met, or the independent choice is neglected, or the product quality cannot be traced. In summary, the reasons for the reluctance to collaborate can be explored in such aspects as the profit, product quality, efficiency, trust and security and eventually some countermeasures should be taken accordingly. Hence, a manufacturing service collaboration solution, which is secure, trustworthy, and multiple users satisfied, is the biggest challenge for manufacturing service collaboration under the Industrial Internet platform.

Manufacturing service collaboration can be realized efficiently based on the process of manufacturing service optimalselection, composition, as well as scheduling. Participants who provide, use or manage the manufacturing services can work together for a common goal (Tao, Zhang, Liu, Cheng, Wang, \& Xu, 2015). QoS (Quality of Service) properties (e.g. response time, lead time, availability, price and reliability) are the most frequently considerations in manufacturing service collaboration works. Tao et al. have investigated several QoS aware service composition optimal-selection and scheduling methods (Tao, Laili, Xu, \& Zhang, 2013; Tao, Zhao, Hu, \& Zhou, 2009). Que et al. proposed a new information entropy immune genetic algorithm (IEIGA) to solve the manufacturing service composition optimal selection problem considering four QoS indexes (time, reliability, cost, and ability) (Que, Zhong, Chen, Chen, \& Ji, 2018). Jin et al. established a correlation-aware manufacturing service model to characterize the QoS dependence (Jin, Yao, \& Chen, 2017). Aiming at the mass customized production, a customized QoS evaluation model and corresponding method to deal with the fluctuated service requirement are designed (Xue, Liu, \& Wang, 2016). The QoS-aware service composition problem is formulated to a multi-objective optimization model, in which either QoS performance or QoS risk is the individual optimization objective. And an efficient epsilon-dominance multi-objective evolutionary algorithm is developed to solve it (Chen, Dou, Li, \& Wu, 
2016). Based on the usage data from previous retrievals of past service users, the missing QoS values for an active service user can be predicted to make personalized service recommendation (Zhang, Zhang, Chen, \& Pan, 2013). According to consumers' preferences and demands, which are also the criterions that the QoS properties should meet, personalized manufacturing service compositions are provided. Especially, the consumer requirements evaluation and risk management in industrial product service systems are investigated for service scheduling (Song, Ming, Han, \& Wu, 2013; Zheng, Ming, Li, \& He, 2015). Meanwhile, transportation (Akbaripour, Houshmand, Woensel, \& Mutlu, 2017; Wang, Zhang, Tao, Chen, Cheng, \& Yang, 2018; Xu, 2015; Zhang, Yang, Zhang, Yu, \& Chen, 2018) and energy consumption (Tao, Bi, Zuo, \& Nee, 2017; Tao, Bi, Zuo, \& Nee, 2016; Xiang, Hu, Yu, \& Wu, 2014; J. Zhou \& Yao, 2017; Zhu, Yang, Chen, Wang, Yin, \& Liu, 2014) have also attracted much more attentions. In addition, the profit for manufacturing service providers are also considered. A pricing model to maximize profit within the satisfactory level of service consumer has been established (Lee, Wang, Zomaya, \& Zhou, 2012). A distributed online approach to schedule and route multicast-oriented tasks for maximizing the time-average profit is proposed $(\mathrm{Wu}, \mathrm{Lu}, \& \mathrm{Zhu}, 2016)$. The obtained scheduling solution can guarantee user-level QoS and improve IaaS providers' credibility and economic benefit (Zuo, Zhang, \& Tan, 2014). Considering the benefit of the two or three sides of the users (provider, consumer and operator), the comprehensive utility models are established (Cheng, Tao, Liu, Zhao, Zhang, \& Xu, 2013; Liu, Zhang, Tao, \& Wang, 2017; Tao, Cheng, Zhang, \& Zhao, 2012). As for manufacturing service scheduling, multiple methods and strategies have been proposed to solve the dynamic and large-scale tasks. Four scheduling strategies for 3D managed print service of varying scales are investigated (Gopsill \& Hicks, 2018). An agent-based scheduling mechanism is designed to allocate real-time tasks and dynamically provision resources (Zhu, Chen, Yang, \& Xiang, 2015). In order to maximize task scheduling performance and minimize nonreasonable task allocation, a dynamic cloud task scheduling based on a two-stage strategy is proposed (Zhang, \& Zhou, 2017). To solve the task scheduling problem in the dynamic cloud manufacturing environment with randomly arriving tasks, an event-triggered dynamic task scheduling method is presented (Zhou, Zhang, Sarker, Laili, \& Ren, 2017).

Based on the illustrations above, manufacturing services are arranged by the operator according to the current demands of the consumers. The participants join the collaboration passively, where the final solution is obtained without common recognition during the collaboration process. In addition, the individual potential intention is always neglected. Hence, collaboration motivation is required considering the individual potential to reach a consensus.

Blockchain, known as a distributed ledger technology, has achieved increasing attention (Feng, He, Zeadally, Khan, \& Kumar, 2018). The secure and tamper-resistant data record manner ensures its widely applications in various domains (Wong, Bhattacharya, \& Butte, 2019). Except the cryptocurrency, blockchain is also employed in industrial environment where emerging data are produced by Internet of Things devices and transactions. As blockchain can provide a fair and transparent data sharing environment, trustworthy platforms and systems for IoT ecosystems and mechanisms are studied (Zhu, Wu, Gai, \& Choo, 2019; Liu, \& Zou, 2019; Hawlitschek, Notheisen, \& Teubner, 2018). Meanwhile, the security and privacy issues existing in distributed system and network are resolved by blockchain technology (Li, Barenji, \& Huang, 2018; Aitzhan, \& Svetinovic, 2018; Li, Kang, Yu, Ye, Deng, \& Zhang, 2018). Blockchain-based mechanism could facilitate data protection and verification without a trusted third-party, and provide end-to-end trust for trading (Yu, Wright, Nepal, Zhu, Liu, \& Ranjan, 2018). Blockchain Platform for Industrial Internet of Things can provide a decentralized, trustless, peer-to-peer network for manufacturing applications (Bahga, \& Madisetti, 2016).

In order to realize a secure and trustworthy manufacturing service collaboration, a blockchain-based mechanism could be utilized to support collaboration among distributed participants to complete trustworthy transactions (Yu, Wright, Nepal, Zhu, Liu \& Ranjan, 2018). In the decentralized blockchain based platform, the group collaboration mechanism, in which multi interest-independent individuals involve, works rather than the operator of platform dominant. Distributed manufacturing service collaboration characterized as the heterogeneity of collaborative participants, the dynamics of collaborative processes, and the inconsistency of collaborative participants' opinions. There should be a suitable collaboration mechanism that allows participants to communicate with each other and guide them to a consensus. Therefore, multi-users satisfied manufacturing service collaboration could be realized by group consensus. Based on the establishment of the underlying data and network layer of the blockchain, the collaboration strategy of manufacturing services based on group consensus is analyzed.

The rest of this paper is organized as follows. In Section 2, the mathematic model of the manufacturing service collaboration optimization problem is presented. Section 3 discusses the proposed method as well as its executive process. An improved Memetic algorithm (MA) is proposed in Section 4. Section 5 gives experimental results and verifies the feasibility of the proposed collaboration method. Finally, Section 6 draws the conclusions and indicates future work.

\section{Problem Formulation}

\subsection{Problem Statement}

Distributed manufacturing services converge on the Industrial Internet platform for multiple users. There are primary three kinds of participants (i.e., provider, consumer and operator) in manufacturing service collaboration, who are selfinterested. The providers publish the shared manufacturing services for the purpose of collaboration in the Industrial Internet platform. The consumers submit their tasks and look for collaborators to accomplish the tasks. The operator of the 
platform manages the services, tasks and transaction processes. Manufacturing service collaboration realizes the collaboration among different participants. Therefore, individual demand satisfaction boosts the passion of collaboration.

The arrival time of the manufacturing tasks are uncertain, and the execution time of the tasks are random. Multiple tasks to be processed arrive at the same time, which can be denoted as $T^{t}=\left\{T_{1}, T_{2}, \ldots, T_{i}, \ldots, T_{s}\right\}$, in which $T_{i}=\left\{\left\{s t_{l}\right\}, t_{A}, T^{R e q}\right.$, $\left.C^{R e q}, Q^{R e q}, T y_{q}, P r i_{t a}^{i}\right\}$. Each task can be decomposed to $l$ subtasks $\left\{s t_{l}\right\}$. $t_{A}$ is the arrival time of $T_{i}$. $T^{R e q}$ is the deadline, and $\operatorname{Pri}_{t a}^{i}$ is the priority degree at $t_{A}$. Ty $y_{q}$ represents the type of task $T_{i}$, where $T y_{q}=\left\{T y_{q} \mid 1 \leqslant q \leqslant \mathrm{~m}\right\}$ and any two task types are mutually independent. The function-similar manufacturing services $\left(S_{1}, S_{2}, \ldots, S_{m i}\right)$ corresponding to each kind of subtask will be selected. The subtask with higher priority (Pri $\left.i_{t a}^{i}\right)$ will have the opportunity to insert into the execution queue. The subtask driven manufacturing service collaboration can reduce the waiting time of the whole task completion and arouse the participation enthusiasm of providers.

Long/short-term utility is employed to assess the satisfaction of the participants who join the manufacturing service collaboration. The providers with long-term expectation, who concentrate on both the current and the potential acquisition, pursue the short execution time, high profit and reputation in the following transactions. At the same time, the consumers with short-term expectation seek for the low price, short delivery time and high product quality (Zhang, Tao, Liu, Zhang, Cheng, \& Zuo, 2019). According to the long-term utility of the providers $\left(U^{P}\right)$ and the short-term utility of the consumers ( $\left.U^{C}\right)$, the satisfaction assessment could help select the optimal manufacturing services and arrange them.

Assuming that services are provided by different providers respectively, the nomenclature is defined as shown in Table 1.

Table 1 Nomenclature

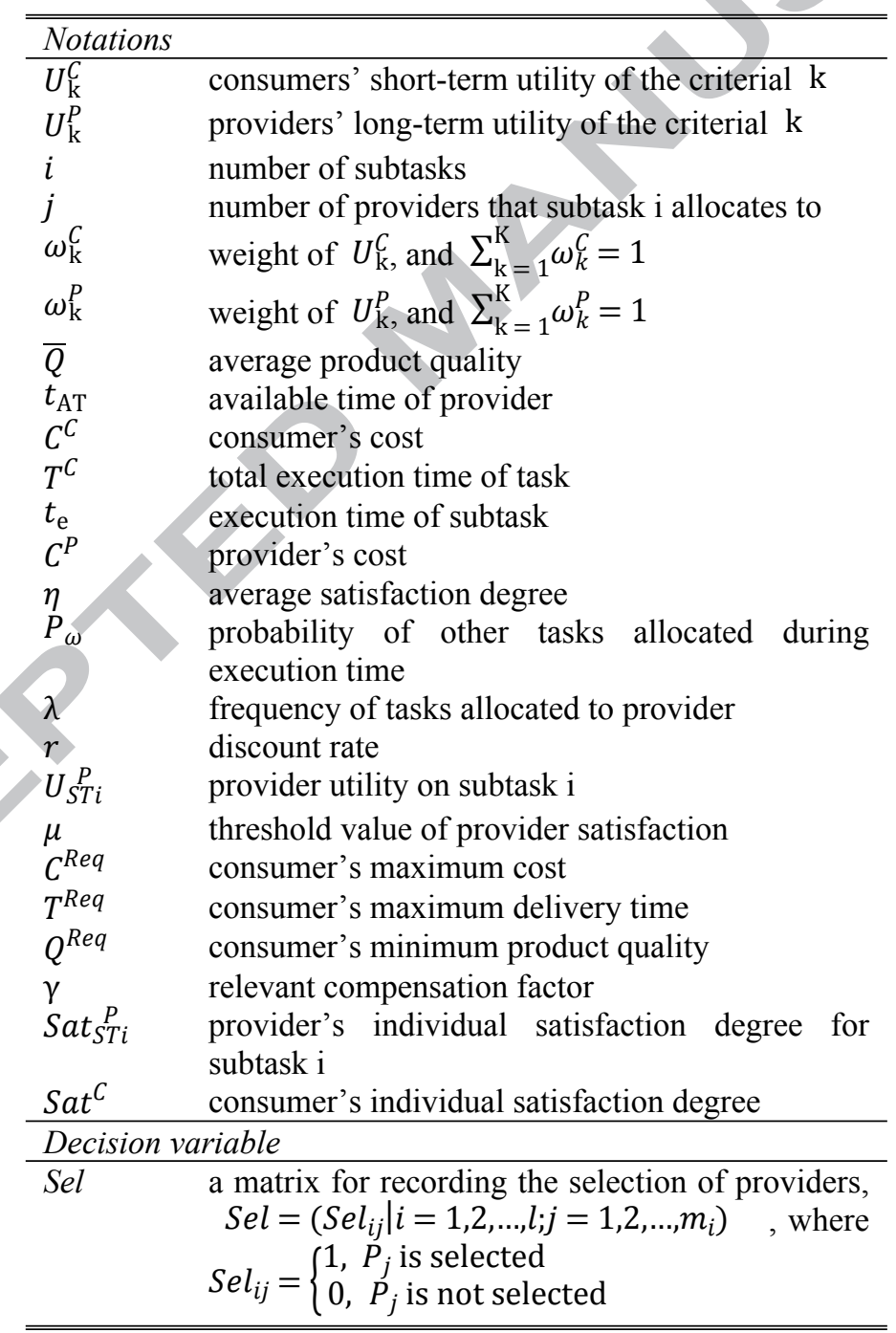

\subsection{Mathematical Model}

\subsubsection{Long/short-term utility modeling of participants}

In order to address the manufacturing service collaboration optimization problem, the following models are used to assess the satisfaction of participants. The comprehensive utility model can be expressed as follows. 
$U=\sum_{\mathrm{k}=1}^{K} \omega_{k} U_{k}$

$U_{k}=e^{-\gamma k}$

Consumers want to get the best service at the lowest price in the current transaction, which can be calculated as the shortterm utility. The properties including execution time, cost and product quality are considered. Mathematically, the shortterm utility for a consumer $\left(U^{C}\right)$ can be expressed as follows.

$U^{C}=\sum_{\mathrm{k}=1}^{K} \omega_{k} U_{k}=\omega_{c}^{C} U_{C}^{C}\left(C^{C}\right)+\omega_{t}^{C} U_{T}^{C}\left(T^{C}\right)+\omega_{Q}^{C} U_{Q}^{C}(\bar{Q})$

$C^{C}=\sum_{i} \sum_{j}\left(\operatorname{Sel}_{i j} * C_{i j}^{C}\right)$

$T^{C}=\sum_{i} \sum_{j}\left(\operatorname{Sel}_{i j} *\left(t e_{\mathrm{ij}}+t_{\mathrm{AT}}\right)\right)$

$\bar{Q}=\frac{1}{l} \sum_{i} \sum_{j}\left(\operatorname{Sel}_{i j} * Q_{i j}\right)$

where $U_{C}^{C}, U_{T}^{C}$ and $U_{Q}^{C}$ are the cost utility, time utility and quality utility, respectively. $\omega_{c}^{C}, \omega_{t}^{C}$ and $\omega_{Q}^{C}$ are utility preferences, which represent the consumer's type, such as cost-sensitive, time sensitive or neither, and $0 \leq \omega_{c}^{C}, \omega_{t}^{C}, \omega_{Q}^{C} \leq 1$.

Providers usually care about not only the current transaction but also the acquisition in the following period, which can be calculated as the long-term utility. The properties including execution time, cost and reputation are considered. Mathematically, the long-term utility for a provider $\left(U^{P}\right)$ can be expressed as follows.

$U^{P}=U_{0}^{P}-U_{1}^{P} * \delta$

$U_{0}^{P}=\sum_{\mathrm{k}=1}^{K} \omega_{k} U_{k}=\omega_{c}^{P} U_{C}^{P}\left(C^{P} * t_{\mathrm{e}}\right)+\omega_{t}^{P} U_{T}^{P}\left(t_{\mathrm{e}}\right)+\omega_{R e p}^{P} U_{R e p}^{P}(\eta)$

$U_{0}^{P}$ is the utility value when the provider accepts the current subtask. And $U_{1}^{P}$ is the utility value of the potential subtask that allocated to the provider during the execution process of the current subtask. As the arrival time of subtasks are uncertain, $U_{1}^{P}$ is approximate as follows.

$U_{1}^{P}=P_{\omega} * U_{0}^{P}$

$P_{\omega}=1-\mathrm{e}^{-\lambda t_{e}}$

$\delta$ is the discount factor, which represents the patience level or the time preference of the provider. The larger the discount factor, the more patient it is, and vice versa. In addition, the discount factor is not constant in reality, and it depends on the time experienced, which has been approximate to the execution time.

$$
\begin{aligned}
& \delta=1 /\left(1+r * t_{e}\right) \\
& U^{P}=\left[\omega_{c}^{P} U_{C}^{P}\left(C^{P}\right)+\omega_{t}^{P} U_{T}^{P}\left(t_{\mathrm{e}}\right)+\omega_{R e p}^{P} U_{R e p}^{P}(\eta)\right] *\left[1-\left(1-\mathrm{e}^{-\lambda t_{e}}\right) * 1 /\left(1+r * t_{e}\right)\right]
\end{aligned}
$$

\subsubsection{Optimization objective and Constraints}

Maximizing consumer's utility value is the fundamental goal of their participation in manufacturing service collaboration. Objective function can be written to maximize the short-term utility of consumers and the long-term utility of providers should be guaranteed. Based on the equation given in (3), the formula of the objective can be written as follows.

$$
\max \left(U^{C}\right)=\omega_{c}^{C} U_{C}^{C}\left(C^{C}\right)+\omega_{t}^{C} U_{T}^{C}\left(T^{C}\right)+\omega_{Q}^{C} U_{Q}^{C}(\bar{Q})
$$

The main constraints are shown as following: 
$C^{C}<C^{R e q}$

$T^{C}<T^{R e q}$

$\bar{Q}>Q^{R e q}$

The first constraint means that the provider's satisfaction degree should be higher than the threshold. The details will be discussed in Section 3. The second constraint shows that total price cannot exceed the budget of consumers. The third constraint indicates that the delivery time is less than the time constraint. The forth constraint represents that the final product quality must be no less than the consumer's request.

\section{Manufacturing service collaboration optimization}

Manufacturing tasks are submitted by personalized consumers randomly on Industrial Internet platform. Providers will receive several collaboration requests at the same time, where they can select from with different satisfaction degrees. In view of the requests that seldom providers willing to accept, the consensus mechanism aware satisfaction promotion can be applied by the operator to stimulate the collaboration process.

\subsection{The executive process of the manufacturing service collaboration optimization}

The executive process of the proposed collaboration method is shown in Fig. 1. The starting point of the method is the tasks submitted by consumers. And the end point of the process is the collaboration plan, in which participants are selected from manufacturing service providers. There are mainly three procedures in the collaboration method, including task processing, service candidates selection and manufacturing service collaboration.

In the procedure of task processing, tasks are submitted to the Industrial Internet platform randomly by consumers. Most of the tasks can be decomposed to several subtasks, which can be completed by manufacturing services respectively. Considering the tasks arriving at the same time, the information of the tasks will be stored including arrival time, deadline, the upper limit of the price, quality degree, priority, etc. at the submit time. In addition, the historical information of the consumers and the tasks have been storied in the blockchain based Industrial Internet platform. The historical information of the consumers' transactions and behaviors could help to better understand the habit and the preference of the consumers.

Manufacturing services owned by providers can perform different kinds of subtasks. The function-similar manufacturing services, whose available time is between the arrival time and the deadline, will be selected as the candidates. The available time means when there is a subtask completion and an opportunity for a new subtask could insert. In order to finish all tasks in a comparative short time, the collaboration process will be triggered by subtasks instead of tasks to reduce the waiting time.

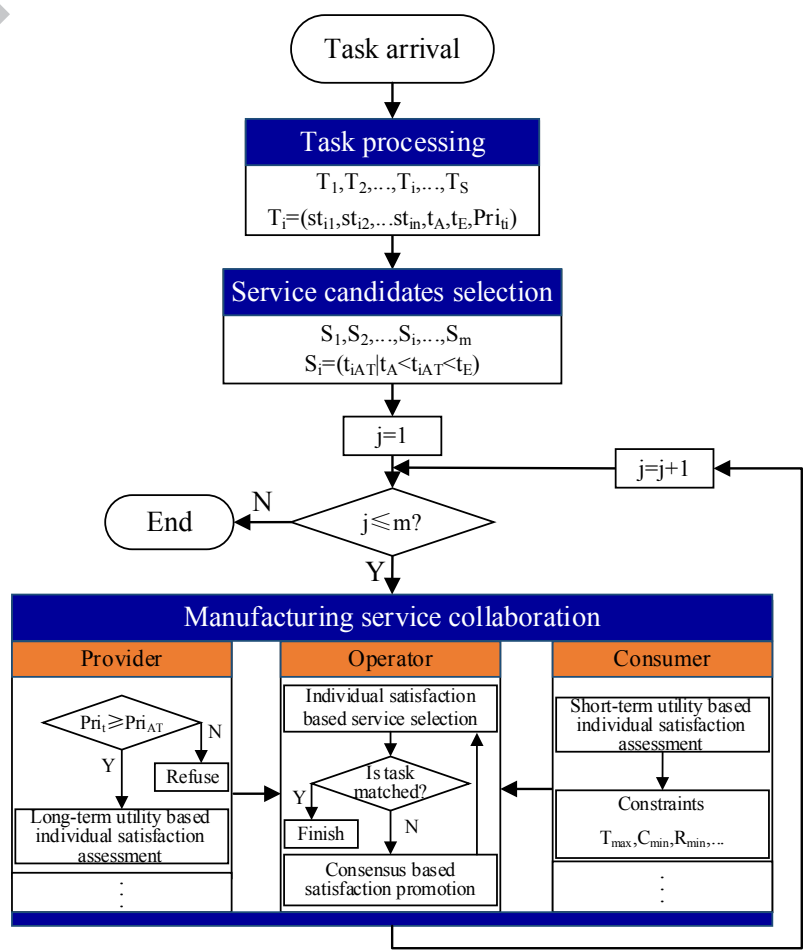


Fig. 1 The executive process of the manufacturing service collaboration optimization

In order to finish the tasks in a willingness manner, the last procedure plays an important role. Three kinds of participants including providers, an operator and consumers are considered. When a provider receives multiple requests, the new subtasks get the chance to insert to the execution queue if the priority of the new subtask is higher than the scheduled subtask. Specific to the subtasks allocated to every provider, the long-term utility based individual satisfaction for each subtask will be calculated. According to the satisfaction degrees of different providers, the operator will choose the provider with the highest value in the acceptable satisfaction value to collaborate. If there is no provider want to finish a subtask, the consensus-based satisfaction promotion mechanism will be conducted by the operator to motivate providers to participate. The provider will be incentivized by the operator and the consumer, where they can raise the price in an acceptable limit. In addition, the consumer's satisfaction degree can be used to evaluate the final collaboration plan.

\subsection{Long/short-term utility based individual satisfaction assessment}

Different providers' attitudes or preferences for a subtask is diverse. According to the long/short-term utility values of providers and consumers, the satisfaction degree can be used to reflect the intentions towards different tasks. In order to assess the satisfactory objectively, the individual satisfaction will be calculated according to the long/short-term utility. A provider's individual satisfaction degree for a subtask allocated to him can be assessed as follows.

$\operatorname{Sat}_{S T i}^{P}=\frac{U_{S T i}^{P}}{M a x U^{P}}$

The individual satisfaction assessment for different subtasks at the same time can reduce the time-related and subjectrelated influences. If the long-term utility based satisfaction degree is less than the acceptable threshold, the provider will refuse to accomplish the subtask.

A consumer's satisfaction degree is associated with the historical transaction satisfaction storied in the platform. A consumer's individual satisfaction degree for a task can be assessed as follows.

$S a t^{C}=\frac{\mathrm{U}^{C}}{U_{\operatorname{Max}}^{C}}$

where $U_{\text {Max }}^{C}$ is searched from the historical database.

The satisfaction degree $S a t_{S T i}^{P}$ and $S a t^{C}$ can be determined within an interval $S_{S a t}^{P}, S_{S T t^{C}} \in[0,1]$.

\subsection{Consensus aware satisfaction promotion mechanism}

If there is no provider with positive and acceptable satisfaction degree specific to a subtask, the consensus mechanism aware satisfaction promotion should be taken. The consensus mechanism in which average satisfaction degree of the selected providers for the other subtasks in the task will be the objective of the promotion result. The satisfaction degree after promotion can be expressed as follows.

$\operatorname{Sat}_{T i}^{P *}\left(C^{P *}\right)=\operatorname{Min}\left(\frac{\sum S^{*} t_{S T i}^{P}}{m}, \mu_{\min }\right)$

where $C^{P *}$ is the cost after promotion, which will be compensated by the operator and the consumer. The consensus mechanism aware satisfaction promotion can motivate providers to participate in the manufacturing service collaboration with the satisfaction that other participants generally achieve.

\section{An improved Memetic algorithm}

This paper proposes a MA that combines genetic algorithm as global search and simulated annealing as a local search strategy. The purpose of manufacturing service collaboration optimization is to maximize the short-term utility of the consumer under the condition of satisfying the providers, in which the manufacturing service collaboration optimization is a NP-hard problem. Numerous providers could work on the same subtask, similar to Flexible Job Shop Scheduling Problem (FJSP) (Edilson, Gabriel, \& Roberto, 2018). Improved algorithms have been proposed to solve the nonlinear equations systems (Liao, Gong, Yan, Wang, \& Hu, 2018; Gong, Wang, Cai, \& Wang, 2018; Gong, Wang, Cai, \& Yang, 2017) and the optimization problems (Shang, Lining, Ling, \& Kai, 2017; Wang, Lai, Wu, Xing, Wang, \& Ishibuchi, 2018; Yi, Xing, Wang, Dong, Vasilakos, Alavi, \& Wang, 2018). The genetic algorithm is one of the best effective solution to the Job Shop Scheduling method, and a satisfactory solution can be obtained (Pezzella, Morganti, \& Ciaschetti, 2008). However, as FJSP is a more complicated problem, more and more scholars are studying the application of genetic algorithm in FJSP in recent years. Hinai et al. used a two-stage hybrid GA to generate a feasibility scheduling arrangement (Al-Hinai \& Elmekkawy, 2011). Chang et al. proposed a coding method that divides chromosome coding into operation assignment and machine 
selection, which are respectively used with different crossover operators and mutation operators (Chang, Tsai, \& Liu, 2014). Many scholars believe that the MA algorithm has good global search ability and local search ability, which is very suitable for solving FJSP and has achieved good results. Yuan et al. proposed an MA algorithm combining local search with NSGAII to solve the multi-objective FJSP problem. The results show that the results obtained by MA are better (Yuan, \& Xu, 2015). The MA algorithm proposed by Ma et al. introduces two efficient local searchers on the basis of GA to increase the local search ability (Ma, Zuo, Zeng, Liang, \& Jiao, 2014).

However, unlike FJSP, providers in manufacturing service collaboration optimization problem has the right to choose tasks according to its own satisfaction, while the machines in FJSP cannot. This paper proposes a MA that combines genetic algorithm and simulated annealing as a local search strategy for the optimization problem studied in this paper. In addition, the operators such as crossover and mutation in the genetic algorithm are improved in genetic algorithm framework (Watanabe, Ida, \& Gen, 2005).

\subsection{Coding and decoding}

The coding of a chromosome consists of two parts, as shown in Fig.2. The first part contains information about the subtasks used to determine the order in which the subtasks are processed. The second part is the information that the provider determines the assignment plan for each subtask allocated to the provider. Each chromosome represents a task assignment scheme.

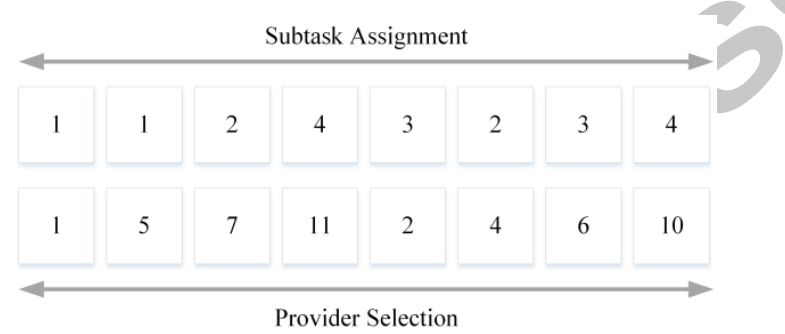

Fig. 2 Chromosome coding scheme

In the first part, the number of genes in the chromosome is equal to the total number of subtasks. The execution order of the subtasks in each task is represented by the task number, and the times of the task number appears is equal to the total number of subtasks. Scan the chromosome from left to right, the kth occurrence of the task number, representing the kth

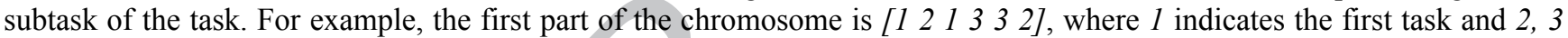
have the same meaning. The 1 in the first position represents the first subtask of the first task, and the 1 in the third position represents the second subtask of the first task.

In the second part, the gene length is the same as the first part and corresponds to the arrangement of the first part. The number represents the provider of the $k t h$ subtask of the $n t h$ task. If the second part of the chromosome corresponding to the first part is [ $\left[\begin{array}{lllllll}2 & 3 & 1 & 3 & 3 & 4\end{array}\right], 2$ in the first position indicates that the first subtask of the first task is assigned to the second provider. 1 in the third position indicates that the second subtask of the first subtask is assigned to the first provider. All providers selected by each subtask form a set $S_{j}$. For example, the first subtask of the first task can be solved by the three providers of 2, 5, and 9 , that is, $S_{1}=\{2,5,9\} . S j$ forms a set $\left\{S_{1}, S_{2}, \cdots \cdots S_{j}\right\}$.

When decoding, the provider that completes each subtask is first determined according to the second part, and then the execution order of the subtasks on the provider was determined by the coding of the first part. They combine the priorities of the tasks to form a complete active scheduling scheme.

\subsection{Crossover operator}

It is important to ensure that the solution after the intersection is still a feasible solution that meets the constraints. The crossover operator of this problem is different from the traditional genetic algorithm. The two parts of the chromosome adopt different crossover methods. The first part adopts POX crossover and the second part adopts multi-point cross method. 


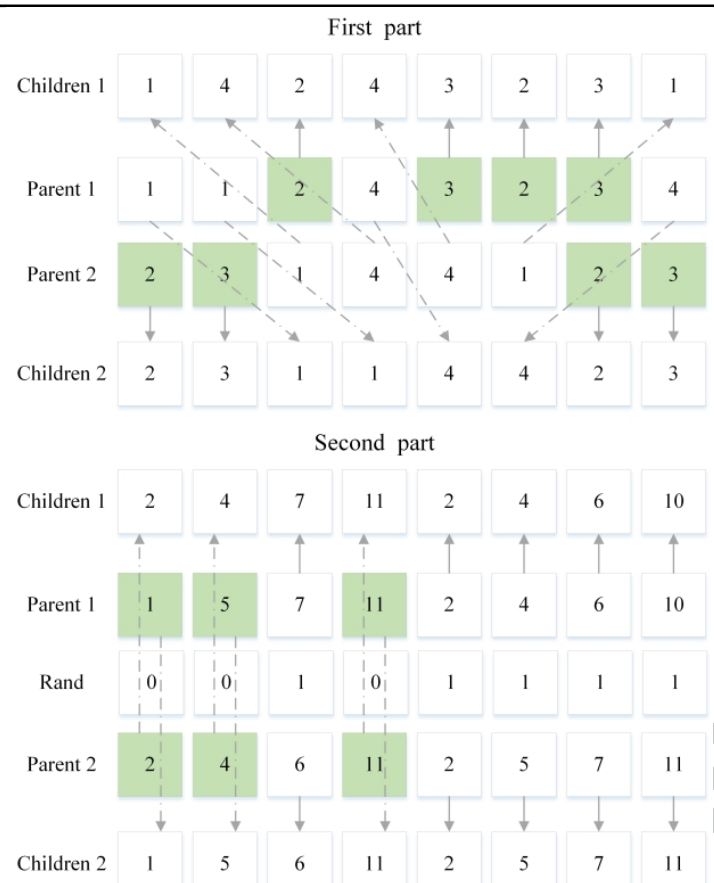

Fig. 3 Crossover operator scheme

In the first part, the task is randomly divided into two categories, $N 1$ and $N 2$. The $N 1$ class retains the original position and inherits to the child, and the $N 2$ class inserts the blanks in order. As shown in Fig. 3, in the first part, the task is randomly divided into two parts, $N 1=\{2,3\}$ and $N 2=\{1,4\}$. Children 1 and Children 2 inherit all the genes and corresponding positions of the N1 class of Parent 1 and Parent 2, respectively. The N2 class of Parent 1 and Parent 2 are sequentially inserted into Children 2 and Children 1 in order. In the second part, the multi-point intersection method is employed. A set of $\{0,1\}$ of the same length as the second part of the chromosome is randomly generated. At the position of 0 , the Parent 1 and Parent 2 corresponding position genes are interchanged.

\subsection{Mutation operator}

In order to avoid assigning tasks to infeasible providers, the mutation operator has been improved from the traditional mutation operator. As shown in Fig.4, in the first part, mutations are made by exchanging genes at two positions on the chromosome. In the second part, the variation uses a random selection of a provider from the set $S j$. For example, $S j=\{1,3,5\}$, the gene is changed from 1 to 3 , which means that the provider has changed from provider 1 to provider 3 .

First part

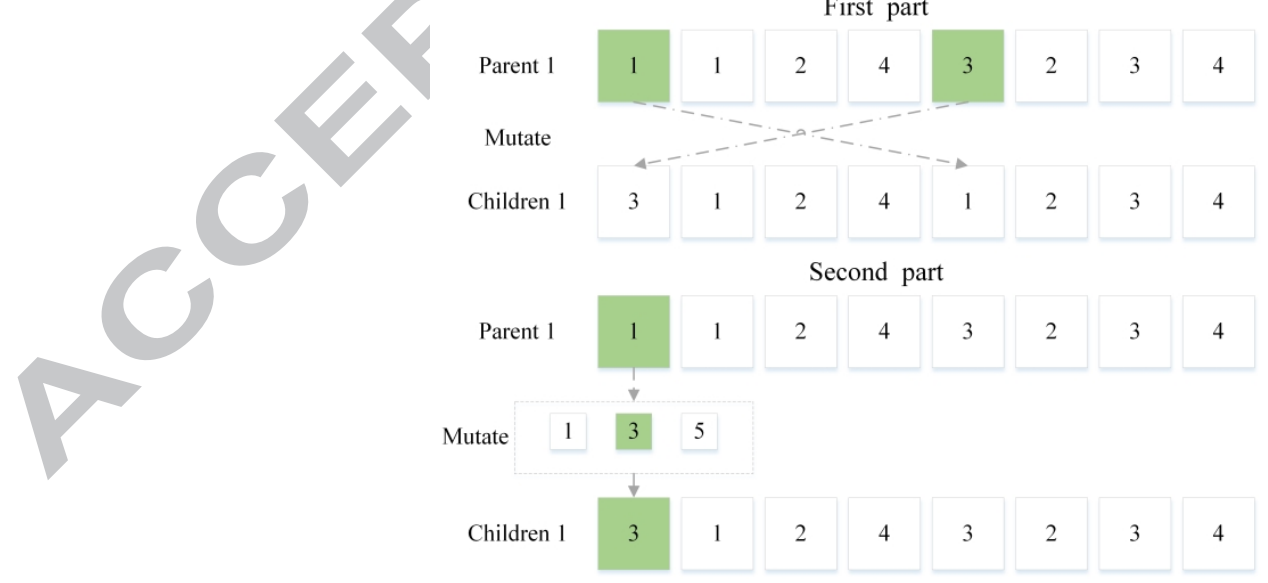

Fig.4 Mutation operator scheme

\subsection{Local search}

Genetic algorithm is easy to fall into "local optimum", so it needs to be combined with local search to enhance local search ability. The genetic algorithm that combines with simulated annealing algorithm is proposed. The simulated annealing process is as follows: 


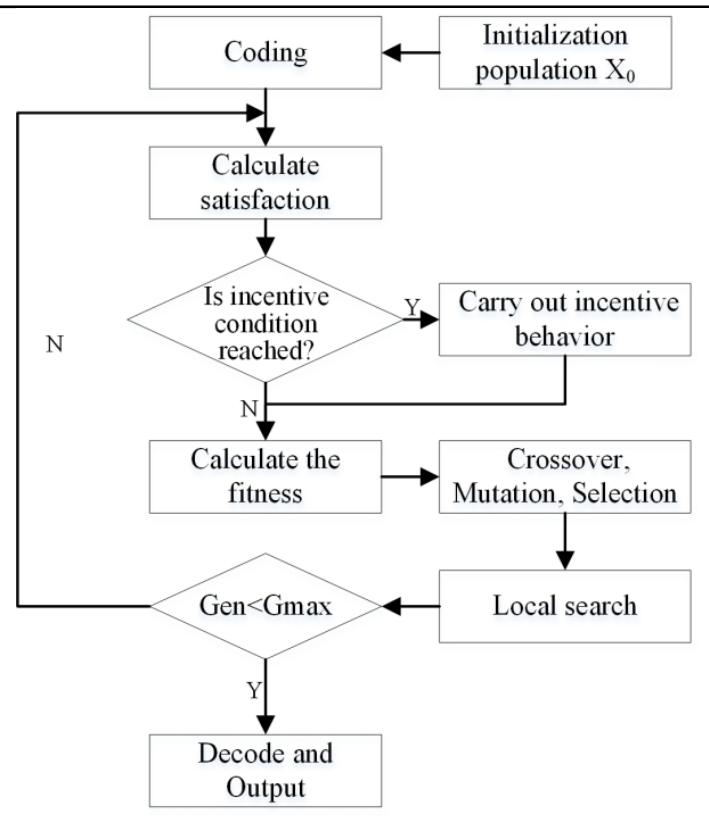

Fig. 5 The flow chart of the proposed algorithm

Step 1. Initializing the annealing temperature $T_{k}(k=0)$, generating an initial solution $X_{0}$;

Step 2. Repeat the following operations for a certain number of times at temperature $T_{k}$ until the stop condition is met:

(1) Randomly select a new feasible solution $X^{\prime}$ in the neighborhood of $X_{0}$. The neighborhood structure is randomly generated by one of the three methods: swap, reversion, and insertion;

(2) Calculate the new fitness of objective function $f\left(X^{\prime}\right)$ to obtain the difference value $\Delta f=f\left(X^{\prime}\right)-f\left(X_{0}\right)$;

(3) If $\Delta f>0$, accept the new solution $X^{\prime}$. Otherwise, the new solution $X^{\prime}$ is accepted when $\min \left\{1, \exp \left(\Delta f / T_{k}\right)\right\}>\operatorname{random}$ $[0,1]$. If a new solution is accepted, then $X_{0}=X^{\prime}$;

(4) Repeat step (1) -(3) until the stop condition is met;

Step 3. Slowly lower the temperature $T$, and repeat Step 1 and Step 2 until the termination condition is satisfied.

The flow chart of the algorithm is shown in Fig. 5

Step 1. Initialization generates population $P_{0}$ and coding.

Step 2. Calculate satisfaction. If the incentive condition of consensus mechanism is reached, the incentive behavior is carried out. If not, step 3 will be carried out directly.

Step 3. Calculate the fitness of the objective function.

Step 4. Perform crossover, mutation and selection operations. The selection operator adopts roulette gambling method.

Step 5. Local search using simulated annealing algorithm

Step 6. Repeat Step 2-5 until the iteration termination condition is satisfied, decode and output the scheduling scheme.

\section{Case study}

\subsection{Problem description}

In order to verify the feasibility of the proposed collaboration method, 3D printing tasks are taken as a case for addressing the manufacturing service collaboration optimization problem. 3D printing bridges the gap between creative ideas and tangible object. Widespread adoption of 3D printing is hindered by dominant factors including unaffordable 3D printers, few materials species, difficult providers seeking, etc (Mai, Zhang, Tao, \& Ren, 2016). A variety of on-demand 3D printing services are now emerging on a 3D service platform, which can serve consumers a range of services to fulfill their vision. 3D printing service collaboration optimization could help gathering appropriate providers with long/short-term utility satisfaction respectively. There are mainly four types of 3D printing requirements on the platform, including image/text requirement, 3D model requirement, object reverse requirement and hybrid requirement. Table 2 shows the task type and sequence in detail.

Table 2 Task type and sequence

\begin{tabular}{|c|c|c|c|c|c|c|}
\hline \multirow[b]{2}{*}{ ID } & \multirow[b]{2}{*}{ Task type } & \multicolumn{5}{|c|}{ Subtask sequence } \\
\hline & & $\begin{array}{c}\text { Model } \\
\text { construction }\end{array}$ & $\begin{array}{c}\text { Model } \\
\text { checking }\end{array}$ & 3D scanning & 3D printing & $\begin{array}{c}\text { Post } \\
\text { processing }\end{array}$ \\
\hline 1 & $\begin{array}{c}\text { Image } \\
\text { /text }\end{array}$ & (1) & (2) & $x$ & (3) & (4) \\
\hline 2 & 3D model & $x$ & (1) & $x$ & (2) & (3) \\
\hline 3 & Object & $x$ & $x$ & (1) & (2) & (3) \\
\hline
\end{tabular}


reverse

$4 \quad$ Hybrid

(1)

(2)

(3)

(4)

(5)

Table 3 Provider candidates of each subtask

\begin{tabular}{llllll}
\hline \hline \multirow{2}{*}{ Task ID } & \multicolumn{5}{c}{ Provider candidates } \\
\cline { 2 - 6 } & MCon & MChe & Scan & Print & Proc \\
\hline 1 & $\mathrm{P}_{1} / \mathrm{P}_{2} / \mathrm{P}_{3} / \mathrm{P}_{4}$ & $\mathrm{P}_{4} / \mathrm{P}_{5}$ & $/$ & $\mathrm{P}_{1} / \mathrm{P}_{7} / \mathrm{P}_{9}$ & $\mathrm{P}_{6} / \mathrm{P}_{10} / \mathrm{P}_{11}$ \\
2 & $/$ & $\mathrm{P}_{4} / \mathrm{P}_{5}$ & $/$ & $\mathrm{P}_{1} / \mathrm{P}_{9}$ & $\mathrm{P}_{6} / \mathrm{P}_{10}$ \\
3 & $/$ & $\mathrm{P}_{4} / \mathrm{P}_{5}$ & $/$ & $\mathrm{P}_{1} / \mathrm{P}_{7} / \mathrm{P}_{8} / \mathrm{P}_{9}$ & $\mathrm{P}_{6} / \mathrm{P}_{10} / \mathrm{P}_{11}$ \\
4 & $/$ & $/$ & $\mathrm{P}_{6}$ & $\mathrm{P}_{1} / \mathrm{P}_{7} / \mathrm{P}_{8} / \mathrm{P}_{9}$ & $\mathrm{P}_{6} / \mathrm{P}_{10} / \mathrm{P}_{11}$ \\
5 & $\mathrm{P}_{1} / \mathrm{P}_{3} / \mathrm{P}_{4}$ & $\mathrm{P}_{4}$ & $/$ & $\mathrm{P}_{1} / \mathrm{P}_{7} / \mathrm{P}_{9}$ & $\mathrm{P}_{6} / \mathrm{P}_{10}$ \\
6 & $/$ & $\mathrm{P}_{4}$ & $/$ & $\mathrm{P}_{1} / \mathrm{P}_{7} / \mathrm{P}_{9}$ & $\mathrm{P}_{6} / \mathrm{P}_{10}$ \\
7 & $/$ & $/$ & $\mathrm{P}_{6}$ & $\mathrm{P}_{1} / \mathrm{P}_{7} / \mathrm{P}_{9}$ & $\mathrm{P}_{6} / \mathrm{P}_{10} / \mathrm{P}_{11}$ \\
8 & $\mathrm{P}_{1} / \mathrm{P}_{2} / \mathrm{P}_{3}$ & $\mathrm{P}_{4} / \mathrm{P}_{5}$ & $/$ & $\mathrm{P}_{7} / \mathrm{P}_{8} / \mathrm{P}_{9}$ & $\mathrm{P}_{10} / \mathrm{P}_{11}$ \\
9 & $/$ & $\mathrm{P}_{4} / \mathrm{P}_{5}$ & $/$ & $\mathrm{P}_{7} / \mathrm{P}_{8} / \mathrm{P}_{9}$ & $\mathrm{P}_{10} / \mathrm{P}_{11}$ \\
10 & $/$ & $/$ & $\mathrm{P}_{6}$ & $\mathrm{P}_{7} / \mathrm{P}_{9}$ & $\mathrm{P}_{10} / \mathrm{P}_{11}$ \\
11 & $\mathrm{P}_{1} / \mathrm{P}_{2} / \mathrm{P}_{3}$ & $\mathrm{P}_{4} / \mathrm{P}_{5}$ & $/$ & $\mathrm{P}_{7} / \mathrm{P}_{8} / \mathrm{P}_{9}$ & $\mathrm{P}_{10} / \mathrm{P}_{11}$ \\
12 & $/$ & $\mathrm{P}_{4} / \mathrm{P}_{5}$ & $/$ & $\mathrm{P}_{7} / \mathrm{P}_{8} / \mathrm{P}_{9}$ & $\mathrm{P}_{10} / \mathrm{P}_{11}$ \\
13 & $\mathrm{P}_{1} / \mathrm{P}_{2} / \mathrm{P}_{3}$ & $\mathrm{P}_{4} / \mathrm{P}_{5}$ & $\mathrm{P}_{6}$ & $\mathrm{P}_{7} / \mathrm{P}_{8} / \mathrm{P}_{9}$ & $\mathrm{P}_{10} / \mathrm{P}_{11}$ \\
14 & $/$ & $/$ & $\mathrm{P}_{6}$ & $\mathrm{P}_{7} / \mathrm{P}_{9}$ & $\mathrm{P}_{10} / \mathrm{P}_{11}$ \\
15 & $\mathrm{P}_{1} / \mathrm{P}_{2} / \mathrm{P}_{3}$ & $\mathrm{P}_{4} / \mathrm{P}_{5}$ & $/$ & $\mathrm{P}_{7} / \mathrm{P}_{8} / \mathrm{P}_{9}$ & $\mathrm{P}_{10} / \mathrm{P}_{11}$ \\
\hline \hline
\end{tabular}

Considering the tasks submitted at the same time, 15 tasks are generated randomly. The task type of each task is randomly obtained from the 4 given task types. Each task type consists of 3-5 subtasks in sequential, which include the services of model construction (MCon), model checking (MChe), 3D scanning (Scan), 3D printing (Print) and post processing (Proc). Five kinds of manufacturing services are provided by different providers, respectively. The total number of manufacturing service providers is 11 . The provider candidates of each subtask are shown in Table 3 . The generation rules of providers and consumers are shown in Table 4 , and the corresponding information is shown in Table 5 and Table 6.

Table 4 Generation rules of providers and consumers

\begin{tabular}{ll}
\hline \hline Parameter & Value \\
\hline Ty & {$[1,4]$} \\
$t_{a}$ & 0 \\
$T^{R e q}$ & {$[20,90]$} \\
$C^{R e q}$ & {$[50,250]$} \\
$Q^{R e q}$ & {$[0.6,1]$} \\
$P^{R} i_{t a}$ & {$[0,1]$} \\
$\eta$ & {$[0.3,1]$} \\
$t_{e}$ & {$[1,10]$} \\
$t_{\mathrm{AT}}$ & 0 \\
$C^{C}$ & {$[10,55]$} \\
$C^{P}$ & {$[10,50]$} \\
$\mathrm{Q}$ & {$[0.5,1]$} \\
$\mathrm{r}$ & {$[0.5,1]$} \\
$\lambda$ & 0.5 \\
$\mu$ & {$[0.4,0.9]$} \\
$U_{M C x}^{C}$ & {$[0.45,1]$} \\
$\omega_{c}^{P}: \omega_{t}^{P}: \omega_{R e p}^{P}$ & $1: 1: 1$ \\
$\omega_{c}^{C}: \omega_{t}^{C}: \omega_{Q}^{C}$ & $1: 1: 1$ \\
\hline \hline
\end{tabular}

Table 5 Information of consumers

\begin{tabular}{|c|c|c|c|c|c|c|c|c|c|c|c|c|}
\hline \multirow{2}{*}{$\begin{array}{l}\text { Task } \\
\text { ID }\end{array}$} & \multirow{2}{*}{ Ty } & \multirow{2}{*}{$T^{R e q}$} & \multirow{2}{*}{$\operatorname{Pri}_{t a}$} & \multirow{2}{*}{$\eta$} & \multirow{2}{*}{$U_{\operatorname{Max}}^{C}$} & \multirow{2}{*}{$C^{R e q}$} & \multirow{2}{*}{$Q^{\text {Req }}$} & \multicolumn{5}{|c|}{$t_{e}$} \\
\hline & & & & & & & & MCon & MChe & Scan & Print & Proc \\
\hline 1 & 1 & 80 & 0 & 0.45 & 0.51 & 180 & 0.76 & 6 & 1 & 1 & 8 & 8 \\
\hline 2 & 2 & 50 & 0 & 0.33 & 0.70 & 120 & 0.80 & / & 10 & 1 & 6 & 8 \\
\hline
\end{tabular}




\begin{tabular}{lllllllllllll}
\hline 3 & 2 & 55 & 0 & 0.56 & 0.49 & 100 & 0.79 & $/$ & 8 & $/$ & 2 & 8 \\
4 & 3 & 40 & 0 & 0.94 & 0.46 & 150 & 0.82 & $/$ & $/$ & 3 & 5 & 4 \\
5 & 1 & 54 & 0 & 0.43 & 0.67 & 105 & 0.70 & 4 & 5 & $/$ & 3 & 6 \\
6 & 2 & 30 & 0 & 0.36 & 0.58 & 90 & 0.65 & $/$ & 8 & $/$ & 2 & 8 \\
7 & 3 & 20 & 0 & 0.59 & 0.74 & 120 & 0.70 & $/$ & $/$ & 7 & 4 & 3 \\
8 & 1 & 86 & 0 & 0.75 & 0.69 & 140 & 0.75 & 8 & 4 & $/$ & 3 & 6 \\
9 & 2 & 59 & 0 & 0.82 & 0.66 & 200 & 0.85 & $/$ & 6 & $/$ & 4 & 6 \\
10 & 3 & 43 & 0 & 0.51 & 0.66 & 130 & 0.72 & $/$ & $/$ & 5 & 7 & 2 \\
11 & 1 & 60 & 1 & 0.92 & 0.62 & 180 & 0.78 & 7 & 7 & $/$ & 3 & 1 \\
12 & 2 & 54 & 0 & 0.86 & 0.46 & 128 & 0.85 & $/$ & 9 & $/$ & 9 & 3 \\
13 & 4 & 76 & 0 & 0.61 & 0.55 & 220 & 0.77 & 4 & 4 & 1 & 1 & 6 \\
14 & 3 & 52 & 0 & 0.59 & 0.53 & 132 & 0.67 & $/$ & $/$ & 2 & 4 & 6 \\
15 & 1 & 89 & 0 & 0.84 & 0.87 & 167 & 0.69 & 6 & 8 & $/$ & 8 & 4 \\
\hline \hline
\end{tabular}

Table 6 Information of providers

\begin{tabular}{cccccccc}
\hline \hline Provider & $t_{A T}$ & $C^{C}$ & $C^{P}$ & $\mathrm{Q}$ & $\mathrm{r}$ & $\lambda$ & $\mu$ \\
\hline$P_{1}$ & 0 & 45.64 & 36.75 & 0.82 & 0.52 & 0.5 & 0.75 \\
$P_{2}$ & 0 & 48.93 & 40.58 & 0.62 & 0.99 & 0.5 & 0.80 \\
$P_{3}$ & 0 & 29.29 & 24.15 & 0.59 & 0.99 & 0.5 & 0.80 \\
$P_{4}$ & 0 & 20.63 & 15.05 & 0.64 & 0.53 & 0.5 & 0.70 \\
$P_{5}$ & 0 & 23.55 & 17.97 & 0.99 & 0.71 & 0.5 & 0.60 \\
$P_{6}$ & 0 & 35.43 & 30.06 & 0.84 & 0.61 & 0.5 & 0.82 \\
$P_{7}$ & 0 & 29.08 & 19.25 & 0.82 & 0.82 & 0.5 & 0.73 \\
$P_{8}$ & 0 & 35.58 & 25.63 & 0.96 & 0.58 & 0.5 & 0.87 \\
$P_{9}$ & 0 & 18.14 & 10.89 & 0.68 & 0.80 & 0.5 & 0.70 \\
$P_{10}$ & 0 & 36.92 & 30.78 & 0.81 & 0.74 & 0.5 & 0.60 \\
$P_{11}$ & 0 & 34.31 & 24.64 & 0.74 & 0.82 & 0.5 & 0.71 \\
\hline \hline
\end{tabular}

\subsection{Results and Analysis}

\subsubsection{Results of the Case}

Gantt chart is used to show the manufacturing service collaboration plan. The code is programmed in MATLAB R2014b. All experiments are carried out in the computer with 3.3GHZ CPU and 4G RAM under Windows 10 system. As shown in Fig. 6, the execution time of each kinds of providers are distributed uniformly. The total task completion time is 66 . The proposed method implements the reasonable collaboration to meet the consumer requirements while maximizing the satisfaction of the providers.

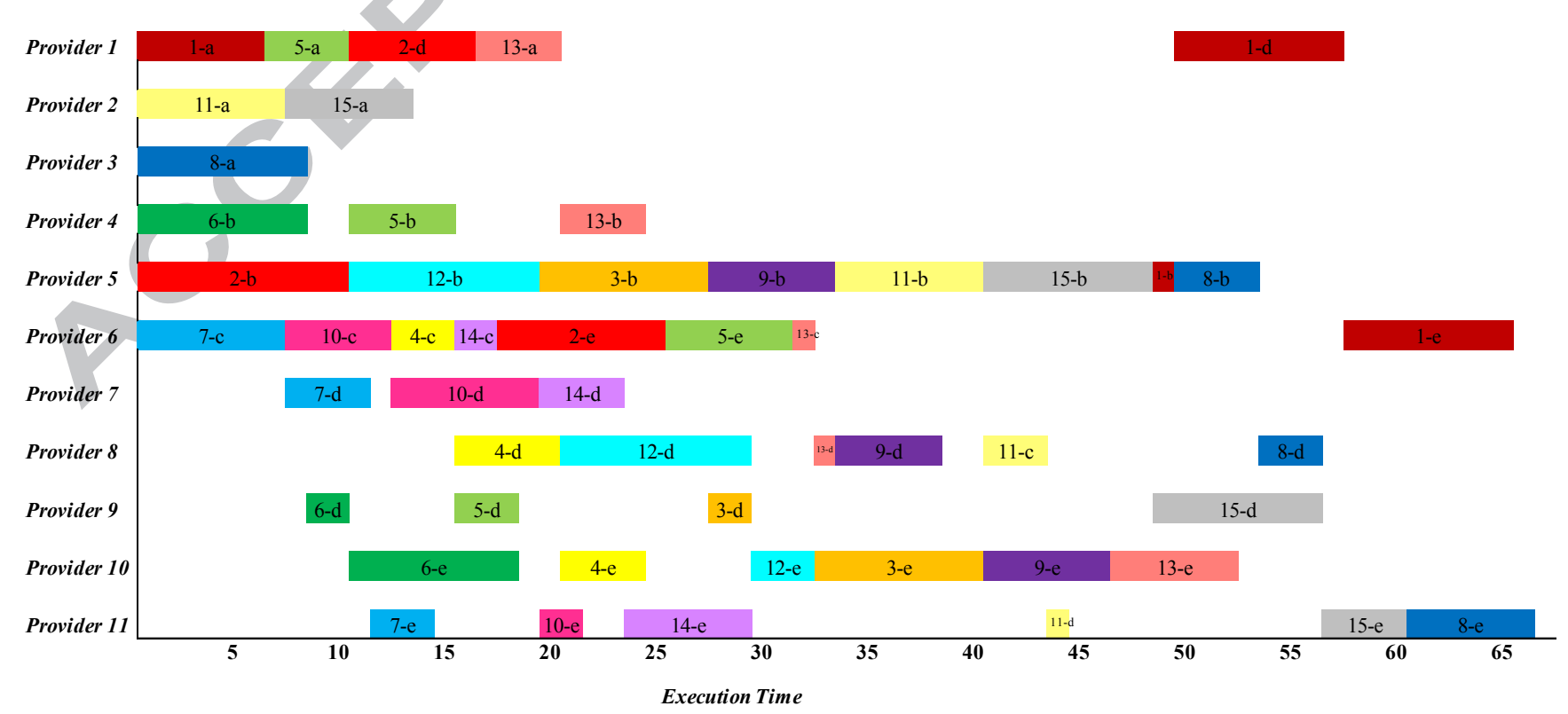

Fig. 6 Manufacturing service collaboration plan

The manufacturing service collaboration optimization solution is shown in Table 7. The cost, delivery time and product quality are also listed, in which the values all meet the consumers' requirements. According to equation (19), the satisfaction degrees of consumers are calculated as shown in Table 7, which are all higher than 0.6. Sat ${ }^{C}$ value greater than 
1 indicates that the short-term utility exceeds the highest historical satisfaction. The priority of the tasks, the satisfaction of the providers, and the length of the waiting queue are all considered when making the manufacturing service collaboration plan. In the process of the manufacturing service collaboration optimization, the threshold value of $P_{6}$ 's satisfaction is 0.82 , while the satisfaction degree on task 10 is 0.81 . In order to achieve satisfactory collaboration, the consensus-based satisfaction promotion mechanism is applied. $C^{P *}$ is calculated according to equation (20). To reach the consensus-based satisfaction value, the operator and the consumer will add 10 yuan as financial compensation to the provider to meet the collaboration qualification.

Table 7 Manufacturing service collaboration optimization solution

\begin{tabular}{llllllllll}
\hline \hline Task & \multirow{2}{*}{$\mathrm{C}^{C}$} & \multirow{2}{*}{$T^{C}$} & \multirow{2}{*}{$\mathrm{Q}^{C}$} & \multirow{2}{*}{$\mathrm{Sat}^{C}$} & \multicolumn{5}{c}{ Providers selection } \\
\cline { 6 - 10 } & & & & & MCon & MChe & Scan & Print & Proc \\
\hline 1 & 150.3 & 65 & 0.87 & 1.13 & $\mathrm{P}_{1}$ & $\mathrm{P}_{5}$ & $/$ & $\mathrm{P}_{1}$ & $\mathrm{P}_{6}$ \\
2 & 104.6 & 25 & 0.88 & 0.89 & $/$ & $\mathrm{P}_{5}$ & $/$ & $\mathrm{P}_{1}$ & $\mathrm{P}_{6}$ \\
3 & 78.6 & 40 & 0.83 & 1.20 & $/$ & $\mathrm{P}_{5}$ & $/$ & $\mathrm{P}_{9}$ & $\mathrm{P}_{10}$ \\
4 & 107.9 & 24 & 0.87 & 1.34 & $/$ & $/$ & $\mathrm{P}_{6}$ & $\mathrm{P}_{8}$ & $\mathrm{P}_{10}$ \\
5 & 119.9 & 31 & 0.75 & 0.83 & $\mathrm{P}_{1}$ & $\mathrm{P}_{4}$ & $/$ & $\mathrm{P}_{9}$ & $\mathrm{P}_{6}$ \\
6 & 75.7 & 18 & 0.71 & 1.04 & $/$ & $\mathrm{P}_{4}$ & $/$ & $\mathrm{P}_{9}$ & $\mathrm{P}_{10}$ \\
7 & 98.8 & 14 & 0.81 & 0.89 & $/$ & $/$ & $\mathrm{P}_{6}$ & $\mathrm{P}_{7}$ & $\mathrm{P}_{11}$ \\
8 & 122.7 & 66 & 0.83 & 0.83 & $\mathrm{P}_{3}$ & $\mathrm{P}_{5}$ & $/$ & $\mathrm{P}_{8}$ & $\mathrm{P}_{11}$ \\
9 & 96.0 & 46 & 0.92 & 0.92 & $/$ & $\mathrm{P}_{5}$ & $/$ & $\mathrm{P}_{8}$ & $\mathrm{P}_{10}$ \\
10 & 98.8 & 21 & 0.81 & 0.92 & $/$ & $/$ & $\mathrm{P}_{6}$ & $\mathrm{P}_{7}$ & $\mathrm{P}_{11}$ \\
11 & 142.4 & 44 & 0.84 & 0.92 & $\mathrm{P}_{2}$ & $\mathrm{P}_{5}$ & $/$ & $\mathrm{P}_{8}$ & $\mathrm{P}_{11}$ \\
12 & 96.0 & 32 & 0.92 & 1.35 & $/$ & $\mathrm{P}_{5}$ & $/$ & $\mathrm{P}_{8}$ & $\mathrm{P}_{10}$ \\
13 & 174.2 & 52 & 0.81 & 1.00 & $\mathrm{P}_{1}$ & $\mathrm{P}_{4}$ & $\mathrm{P}_{6}$ & $\mathrm{P}_{8}$ & $\mathrm{P}_{10}$ \\
14 & 98.8 & 29 & 0.81 & 1.10 & $/$ & $/$ & $\mathrm{P}_{6}$ & $\mathrm{P}_{7}$ & $\mathrm{P}_{11}$ \\
15 & 124.9 & 60 & 0.77 & 0.63 & $\mathrm{P}_{2}$ & $\mathrm{P}_{5}$ & $/$ & $\mathrm{P}_{9}$ & $\mathrm{P}_{11}$ \\
\hline \hline
\end{tabular}

The proposed manufacturing service collaboration optimization method selects the providers and consumers according to the satisfaction degree from both sides of them. The solution will meet their preferences more easily. Specific to the tasks with low preference or satisfaction of providers, the providers will be incentivized by the operator and the consumer to reach a consensus. This will greatly improve the collaboration success rate of the platform.

From the perspective of the consumers, all the requirements are satisfied and most of the consumers' satisfaction degree is higher than the average satisfaction degree. For the operator of the Industrial Internet platform, all tasks are completed with the participants satisfied. For the providers, they have been deeply involved in the collaboration process. When multiple tasks arrive at the same time, the providers can calculate their individual satisfaction degrees for each task based on the long-term utility, which reflect the long-term expects of the providers.

\subsubsection{Comparison of GA, GA-HC and MA algorithm}

To prove the effectiveness of the proposed MA, GA is selected for comparison, which is the most popular used algorithms to solve NP-hard problem. In order to better verify the search ability of our proposed MA, we chose the GA-HC algorithm that combines GA and hill climbing method as a comparison. The hill climbing method is a common used algorithm in local search and is a greedy algorithm. It only accepts a new solution when there is an improvement. Each algorithm runs 1000 iterations. The following results are obtained after 10 repetitions. Fig. 7 gives the comparison results of three algorithms. It can be seen that the Fitness value of MA is higher than that of GA-HC and GA. As shown in Fig. 8, MA, GA-HC and GA run 100, 200, 300, 500, 800, and 1000 generations, and each of them runs 10 times. The red line in the figure represents the median of the 10 experimental results, with the top line representing the maximum and the bottom line representing the minimum. The upper and lower sides of the rectangular box represent the upper quartile and the lower quartile. At each generation, the median, maximum, minimum, upper quartile, and lower quartile in the solution obtained by MA are superior than that of GA-HC and GA. In solving the manufacturing service collaboration problem, the solution of the MA algorithm is far superior to the solution obtained by the GA algorithm. 


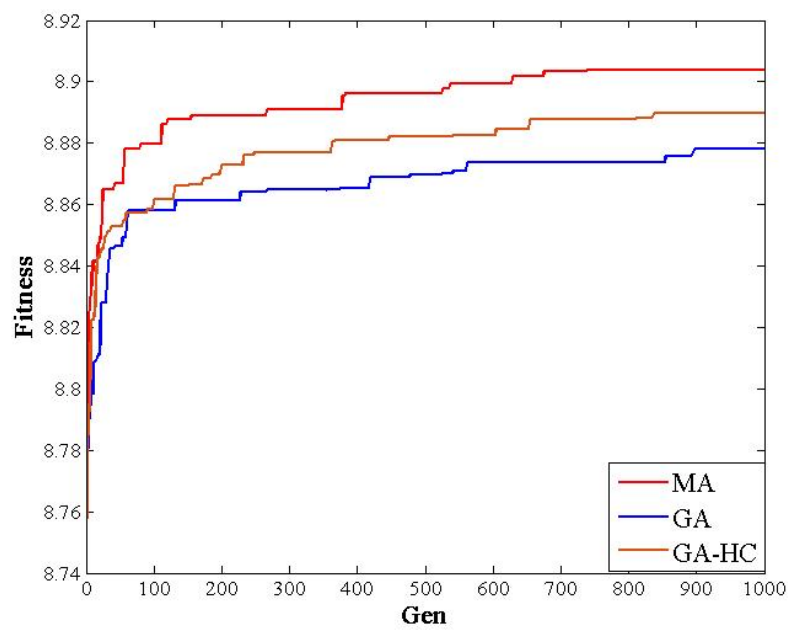

Fig. 7 Comparison of GA, GA-HC and MA algorithm

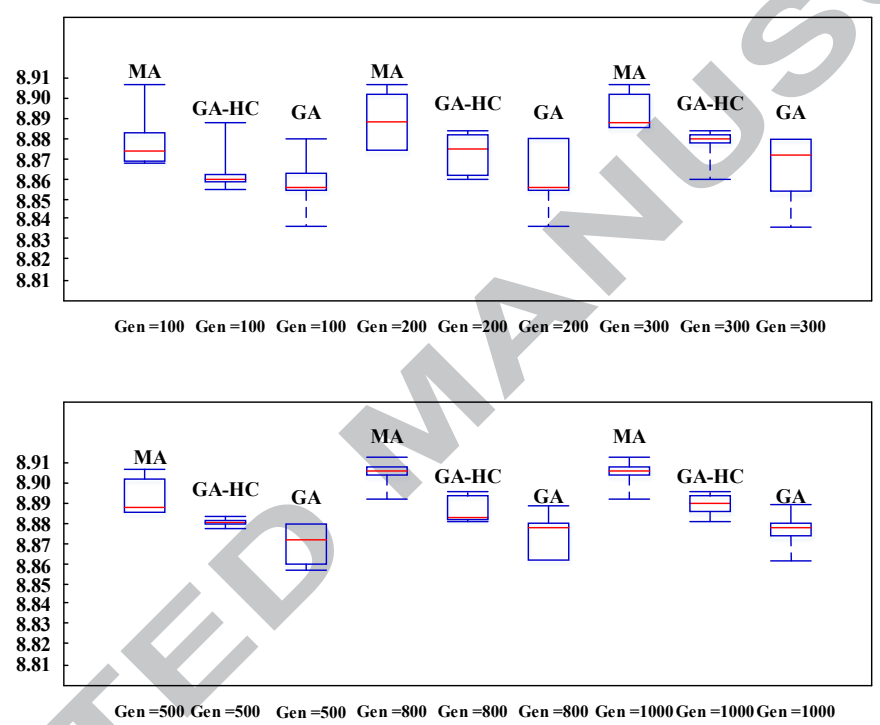

Fig. 8 Box plot of GA, GA-HC and MA algorithm running 10 times

The standard deviation of the 10 solutions obtained by the MA algorithm in these 6 times are $0.0144 .0 .0138,0.0122$, $0.0095,0.0067,0.0067$, respectively. The standard deviation of the 10 solutions obtained by the GA-HC algorithm in these 6 times are $0.0065 .0 .0113,0.0098,0.0030,0.0073,0.0063$, respectively. And the standard deviation of the 10 solutions obtained by the GA algorithm in each generation is $0.0133,0.0158,0.0159,0.0086,0.0102,0.0099$, respectively. It indicates that the MA and GA-HC algorithm is more stable.

The MA algorithm can avoid the "local optimal" problem very well, and it also has good global search ability and local search ability. When the number of iterations is small, the fitness value of the solution obtained by MA is increasing rapidly, and the growth rate is much larger than that of GA-HC and GA.

\section{Conclusions and future works}

In this paper, manufacturing service collaboration optimization problem under a blockchain based Industrial Internet platform has been presented. As the providers are no longer only concentrate on current transactions, they are more concerned about the long-term acquisition, which will help them develop further. The long-term utility of providers and the short-term utility of consumers are analyzed to enhance the manufacturing service collaboration process. The individual satisfaction degree including service time, service cost, product quality and reputation are considered to choose the optimal services for tasks. Based on the platform that could support collaboration among distributed participants to complete trustworthy transactions, the consensus aware collaboration method is proposed to promote the participants' aspiration to collaborate. The approach should be a reference to be applied within a manufacturer between different departments. But it should be analyzed before being used since different departments may not pursue different utilities when they are tight coupling. The collaboration occurs between different uncorrelated manufacturers in the manuscript. The main contributions of this paper are summarized as follows:

(1) For addressing the manufacturing service collaboration optimization problem, the individual satisfaction based on the long/short-term utility model is assessed considering the service time, service cost, product quality and reputation. 
(2) Specific to the tasks with lower satisfaction degree by providers, a consensus-based satisfaction promotion mechanism is proposed to motivate providers to participate in the collaboration. Experimental results have shown that it is effective in solving the problem of low participation willingness for collaboration.

(3) In order to solve the manufacturing service collaboration problem raised in this paper, MA that combines genetic algorithm as global search and simulated annealing as a local search strategy is proposed with incentive mechanism.

However, there are still some details missing in this paper and the possible future work includes: (1) the manufacturing service collaboration optimization problem with large-scale tasks in uncertain arrival time. In actual situation, consumers submit tasks randomly and the quantity of tasks are much larger, which should be further studied. (2) As the collaborators are not all independent of each other in reality, the incidence relations should be considered in collaboration optimization. (3) In addition to the cost incentive method, some other motivation mechanism should be further investigated to promote the manufacturing service collaboration.

\section{References}

Aitzhan, N. Z., \& Svetinovic, D. (2018). Security and privacy in decentralized energy trading through multi-signatures, blockchain and anonymous messaging streams. IEEE Transactions on Dependable and Secure Computing, 15(5), 840-852.

Akbaripour, H., Houshmand, M., Woensel, T. \& Mutlu, N. (2017). Cloud manufacturing service selection optimization and scheduling with transportation considerations: mixed-integer programming models. International Journal of Advanced Manufacturing Technology, (4), 1-28.

Al-Hinai, N. \& Elmekkawy, T.Y. (2011). Robust and stable flexible job shop scheduling with random machine breakdowns using a hybrid genetic algorithm. International Journal of Production Economics, 132(2), 279-291.

Bahga, A., \& Madisetti, V. K. (2016). Blockchain platform for industrial internet of things. Journal of Software Engineering and Applications, 9(10), 533.

Chang, H.C., Tsai, H.T. \& Liu, T.K. (2014). Application of genetic algorithm to optimize unrelated parallel machines of flexible job-shop scheduling problem. IEEE International Conference on Control \& Automation (pp. 596-599).

Chen, F., Dou, R., Li, M., Wu, H. (2016). A flexible QoS-aware Web service composition method by multi-objective optimization in cloud manufacturing. Computers \& Industrial Engineering, 99: 423-431.

Cheng, Y., Tao, F., Liu, Y., Zhao, D., Zhang, L. \& Xu, L. (2013). Energy-aware resource service scheduling based on utility evaluation in cloud manufacturing system. Proceedings of the Institution of Mechanical Engineers Part B Journal of Engineering Manufacture, 227(12), 1901-1915.

Edilson, R. R. K. , Gabriel, D. D. A. A. , \& Roberto, H. T. (2018). A new approach to solve the flexible job shop problem based on an hybrid particle swarm optimization and random-restart hill climbing. Computers \& Industrial Engineering, 178-189

Feng, Q., He, D., Zeadally, S., Khan, M. K., \& Kumar, N. (2018). A survey on privacy protection in blockchain system. Journal of Network and Computer Applications.

Gong, W., Wang, Y., Cai, Z., \& Wang, L. (2018). Finding Multiple Roots of Nonlinear Equation Systems via a RepulsionBased Adaptive Differential Evolution. IEEE Transactions on Systems, Man, and Cybernetics: Systems, (99), 1-15.

Gong, W., Wang, Y., Cai, Z., \& Yang, S. (2017). A weighted biobjective transformation technique for locating multiple optimal solutions of nonlinear equation systems. IEEE Transactions on Evolutionary Computation, 21(5), $697-713$.

Gopsill, J.A. \& Hicks, B.J. (2018). Investigating the effect of scale and scheduling strategies on the productivity of 3D managed print services. Proceedings of the Institution of Mechanical Engineers Part B Journal of Engineering Manufacture, 232(10), 1753-1766.

Hawlitschek, F., Notheisen, B., \& Teubner, T. (2018). The limits of trust-free systems: A literature review on blockchain technology and trust in the sharing economy. Electronic commerce research and applications, 29, 50-63.

Jin, H., Yao, X. \& Chen, Y. (2017). Correlation-aware QoS modeling and manufacturing cloud service composition. Journal of Intelligent Manufacturing, 28(8), 1-14.

Lee, Y.C., Wang, C., Zomaya, A.Y., \& Zhou, B.B. (2012). Profit-driven scheduling for cloud services with data access awareness. Journal of Parallel \& Distributed Computing, 72(4), 591-602.

Li, Z., Barenji, A. V., \& Huang, G. Q. (2018). Toward a blockchain cloud manufacturing system as a peer to peer distributed network platform. Robotics and Computer-Integrated Manufacturing, 54, 133-144.

Li, Z., Kang, J., Yu, R., Ye, D., Deng, Q., \& Zhang, Y. (2018). Consortium blockchain for secure energy trading in industrial internet of things. IEEE transactions on industrial informatics, 14(8), 3690-3700.

Liao, Z., Gong, W., Yan, X., Wang, L., \& Hu, C. (2018). Solving nonlinear equations system with dynamic repulsion-based evolutionary algorithms. IEEE Transactions on Systems, Man, and Cybernetics: Systems, (99), 1-12.

Liu, Q., \& Zou, X. (2019). Research on trust mechanism of cooperation innovation with big data processing based on blockchain. EURASIP Journal on Wireless Communications and Networking, 2019(1), 26.

Liu, Y., Zhang, L., Tao, F. \& Wang, L. (2017). Resource service sharing in cloud manufacturing based on the Gale-Shapley algorithm: advantages and challenge. International Journal of Computer Integrated Manufacturing, 30(4-5), 420432.

Ma, W., Zuo, Y., Zeng, J., Liang, S., \& Jiao, L. (2014, July). A memetic algorithm for solving flexible job-shop scheduling problems. In 2014 IEEE Congress on Evolutionary Computation (CEC) (pp. 66-73). IEEE.

Mai, J., Zhang, L., Tao, F. \& Ren, L. (2016). Customized production based on distributed 3D printing services in cloud manufacturing. International Journal of Advanced Manufacturing Technology, 84(1-4), 1-13. 
Pezzella, F. , Morganti, G., \& Ciaschetti, G. (2008). A Genetic Algorithm for the Flexible Job-Shop Scheduling Problem. Computers and Operations Research, 35(10), 3202-3212.

Pouryazdan, M., Fiandrino, C., Kantarci, B. , Kliazovich, D., Soyata, T. , \& Bouvry, P. (2017). Game-Theoretic Recruitment of Sensing Service Providers for Trustworthy Cloud-Centric Internet-of-Things (IoT) Applications. Globecom Workshops. IEEE Globecom Workshops, pp. 1-6.

Que, Y., Zhong, W., Chen, H., Chen, X. \& Ji, X. (2018). Improved adaptive immune genetic algorithm for optimal QoSaware service composition selection in cloud manufacturing. International Journal of Advanced Manufacturing Technology, 96(9-12), 4455-4465.

Shang, X., Lining, X., Ling, W., \& Kai, Z. (2017). Comprehensive Learning Pigeon-Inspired Optimization with Tabu List. SCIENCE CHINA Technological Sciences, 60(60), 425.

Song, W., Ming, X., Han, Y., \& Wu, Z. (2013). A rough set approach for evaluating vague customer requirement of industrial product-service system. International Journal of Production Research, 51(22), 6681-6701.

Tao, F., Bi, L., Zuo, Y. \& Nee, A.Y.C. (2017). A Cooperative Co-Evolutionary Algorithm for Large-Scale Process Planning With Energy Consideration. Journal of Manufacturing Science and Engineering, 139(6), 061016-061011.

Tao, F., Bi, L.N., Zuo, Y. \& Nee, A.Y.C. (2016). A hybrid group leader algorithm for green material selection with energy consideration in product design. CIRP Annals - Manufacturing Technology, 65(1), 9-12.

Tao, F., Cheng, Y., Xu, L.D., Zhang, L. \& Li, B.H. (2014). CCIoT-CMfg: Cloud Computing and Internet of Things-Based Cloud Manufacturing Service System. IEEE Transactions on Industrial Informatics, 10(2), 1435-1442.

Tao, F., Cheng, Y., Zhang, L. \& Zhao, D. (2012). Utility modelling, equilibrium, and coordination of resource service transaction in service-oriented manufacturing system. Proceedings of the Institution of Mechanical Engineers Part B Journal of Engineering Manufacture, 226(6), 1099-1117.

Tao, F., Laili, Y., Xu, L. \& Zhang, L. (2013). FC-PACO-RM: A Parallel Method for Service Composition OptimalSelection in Cloud Manufacturing System. IEEE Transactions on Industrial Informatics, 9(4), 2023-2033.

Tao, F. \& Qi, Q. (2017). New IT Driven Service-Oriented Smart Manufacturing: Framework and Characteristics. IEEE Transactions on Systems Man \& Cybernetics Systems, PP(99), 1-11.

Tao, F., Zhang, L., Liu, Y., Cheng, Y., Wang, L. \& Xu, X. (2015). Manufacturing Service Management in Cloud Manufacturing: Overview and Future Research Directions. Journal of Manufacturing Science and Engineering, 137(4), 040912-040911.

Tao, F., Zhao, D., Hu, Y. \& Zhou, Z. (2009). Resource Service Composition and Its Optimal-Selection Based on Particle Swarm Optimization in Manufacturing Grid System. IEEE Transactions on Industrial Informatics, 4(4), $315-327$.

Watanabe, M., Ida, K., Gen, M. (2005). A genetic algorithm with modified crossover operator and search area adaptation for the job-shop scheduling problem. Computers \& Industrial Engineering, 48(4), 743-752.

Wang, R., Lai, S., Wu, G., Xing, L., Wang, L., \& Ishibuchi, H. (2018). Multi-clustering via evolutionary multi-objective optimization. Information Sciences, 450, 128-140.

Wang, Y., Zhang, Y., Tao, F., Chen, T., Cheng, Y., \& Yang, S. (2018). Logistics-aware manufacturing service collaboration optimisation towards industrial internet platform. International Journal of Production Research, 1-20.

Wong, D. R., Bhattacharya, S., \& Butte, A. J. (2019). Prototype of running clinical trials in an untrustworthy environment using blockchain. Nature communications, 10(1), 917.

Wu, K., Lu, P. \& Zhu, Z. (2016). Distributed Online Scheduling and Routing of Multicast-Oriented Tasks for Profit-Driven Cloud Computing. IEEE Communications Letters, 20(4), 684-687.

Xiang, F., Hu, Y., Yu, Y. \& Wu, H. (2014). QoS and energy consumption aware service composition and optimal-selection based on Pareto group leader algorithm in cloud manufacturing system. Central European Journal of Operations Research, 22(4), 663-685.

$\mathrm{Xu}, \mathrm{X}$. (2015). Cloud manufacturing service composition based on QoS with geo-perspective transportation using an improved Artificial Bee Colony optimisation algorithm. International Journal of Production Research, 53(14), 4380-4404.

Xue, X., Liu, Z.Z. \& Wang, S.F. (2016). Manufacturing service composition for the mass customised production. International Journal of Computer Integrated Manufacturing, 29(2), 119-135.

Yi, J. H., Xing, L. N., Wang, G. G., Dong, J., Vasilakos, A. V., Alavi, A. H., \& Wang, L. (2018). Behavior of crossover operators in NSGA-III for large-scale optimization problems. Information Sciences, https://doi.org/10.1016/j.ins.2018.10.005.

Yu, B., Wright, J., Nepal, S., Zhu, L., Liu J., \& Ranjan, R. (2018). Iotchain: establishing trust in the internet of things ecosystem using blockchain. IEEE Cloud Computing, 5(4), 12-23.

Yuan, Y., \& Xu, H. (2015). Multiobjective flexible job shop scheduling using memetic algorithms. IEEE Transactions on Automation Science and Engineering, 12(1), 336-353.

Zhang, P.Y., \& Zhou, M.C. (2017). Dynamic Cloud Task Scheduling Based on a Two-Stage Strategy. IEEE Transactions on Automation Science \& Engineering, PP(99), 1-12.

Zhang, W., Yang, Y., Zhang, S., Yu, D. \& Chen, Y. (2018). A new three-dimensional manufacturing service composition method under various structures using improved Flower Pollination Algorithm. Enterprise Information Systems, 12(1), 1-18.

Zhang, W.Y., Zhang, S., Chen, Y.G. \& Pan, X.W. (2013). Combining social network and collaborative filtering for personalised manufacturing service recommendation. International Journal of Production Research, 51(22), 67026719 . 
Zhang, Y., Tao, F., Liu, Y., Zhang, P., Cheng, Y., \& Zuo, Y. (2019). Long/short-term utility aware optimal selection of manufacturing service composition towards Industrial Internet platform. IEEE Transactions on Industrial Informatics, DOI:10.1109/TII.2019.2892777.

Zheng, M., Ming, X., Li, M., \& He, L. (2015). A framework for Industrial Product-Service Systems risk management. Proceedings of the Institution of Mechanical Engineers, Part O: Journal of Risk and Reliability, 229(6), 501-516.

Zhou, J. \& Yao, X. (2017). Multi-objective hybrid artificial bee colony algorithm enhanced with Lévy flight and selfadaption for cloud manufacturing service composition. Applied Intelligence, 47(3), 721-742.

Zhou, L., Zhang, L., Sarker, B.R., Laili, Y. \& Ren, L. (2017). An event-triggered dynamic scheduling method for randomly arriving tasks in cloud manufacturing. International Journal of Computer Integrated Manufacturing, 31(3), 1-16.

Zhu, L., Wu, Y., Gai, K., \& Choo, K. K. R. (2019). Controllable and trustworthy blockchain-based cloud data management. Future Generation Computer Systems, 91, 527-535.

Zhu, X., Chen, C., Yang, L.T. \& Xiang, Y. (2015). ANGEL: Agent-Based Scheduling for Real-Time Tasks in Virtualized Clouds. IEEE Transactions on Computers, 64(12), 3389-3403.

Zhu, X., Yang, L.T., Chen, H., Wang, J., Yin, S. \& Liu, X. (2014). Real-Time Tasks Oriented Energy-Aware Scheduling in Virtualized Clouds. IEEE Transactions on Cloud Computing, 2(2), 168-180.

Zuo, X., Zhang, G., Tan, W. (2014). Self-Adaptive Learning PSO-Based Deadline Constrained Task Scheduling for Hybrid IaaS Cloud. IEEE Transactions on Automation Science \& Engineering, 11(2), 564-573. 


\section{Highlights}

$\checkmark$ The individual satisfaction based on the long/short-term utility is assessed.

$\checkmark$ A consensus-based satisfaction promotion mechanism is proposed for collaboration.

$\checkmark$ Memetic algorithm is used to address MS collaboration optimization problem. 